Cite this: Food Funct., 2014, 5, 1613

Received 12th March 2014 Accepted 12th May 2014

DOI: $10.1039 / c 4 f o 00209 a$

www.rsc.org/foodfunction

\section{Effects of black tea on body composition and metabolic outcomes related to cardiovascular disease risk: a randomized controlled trial $\dagger$}

\author{
Siv K. Bøhn,,$^{\star * a}$ Kevin D. Croft, ${ }^{b}$ Sally Burrows, ${ }^{b}$ Ian B. Puddey, ${ }^{b}$ Theo P. J. Mulder, ${ }^{c}$ \\ Dagmar Fuchs, ${ }^{c}$ Richard J. Woodman ${ }^{d}$ and Jonathan M. Hodgson ${ }^{b}$
}

\begin{abstract}
There is increasing evidence that tea and its non-caffeine components (primarily flavonoids) contribute to cardiovascular health. Randomized controlled trials have shown that tea can improve cardiovascular disease risk factors. We have previously reported a non-caffeine associated beneficial effect of regular black tea consumption on blood pressure and its variation. Objective: To explore the non-caffeine associated effects of black tea on body weight and body fat distribution, and cardiovascular disease related metabolic outcomes. Design: regular tea-drinking men and women ( $n=111 ; \mathrm{BMI} 20-35 \mathrm{~kg} \mathrm{~m}^{-2}$ ) were recruited to a randomized controlled double-blind 6 month parallel-designed trial. Participants consumed 3 cups per day of either powdered black tea solids (tea) or a flavonoid-free flavour- and caffeine-matched placebo (control). Body weight, waist- and hip-circumference, endothelial function and plasma biomarkers were assessed at baseline, 3 months and 6 months. Results: Compared to control, regular ingestion of black tea over 3 months inhibited weight gain $(-0.64 \mathrm{~kg}, p=0.047)$ and reduced waist circumference $(-1.88 \mathrm{~cm}, P=0.035)$ and waist-to-hip ratio $(-0.03, P=0.005)$. These effects were no longer significant at 6 months. There were no significant effects observed on fasting glucose, insulin, plasma lipids or endothelial function. Conclusion: Our study suggests that short-term regular ingestion of black tea over 3 months can improve body weight and body fat distribution, compared to a caffeine-matched control beverage. However, there was no evidence that these effects were sustained beyond 3 months.
\end{abstract}

\section{Introduction}

Besides water, tea is the most popular beverage in the world. In relation to cardiovascular disease (CVD) tea is generally described according to two types: green tea and black tea. Both green and black tea are brewed by infusion of leaves from the tea plant, Camellia sinensis, with boiled water. The brewed beverage contains a wide range of phytochemicals, particularly polyphenols, with potential beneficial bioactivities following consumption. Tea also contains caffeine, about 25 to $50 \mathrm{mg}$ per cup. ${ }^{1}$ Originating from the same plant, green and black tea have overlapping phytochemical profiles. However, differences in product processing of the teas give some distinct differences

${ }^{a}$ Department of Nutrition, Institute of Basic Medical Sciences, Faculty of Medicine, University of Oslo, Norway. E-mail: s.k.bohn@medisin.uio.no; Tel: +4722851516

${ }^{b}$ School of Medicine and Pharmacology, University of Western Australia, WAIMR Centre for Food and Genomic Medicine, Perth, Western Australia, Australia

${ }^{c}$ Unilever Research and Development, Vlaardingen, The Netherlands

${ }^{d}$ Discipline of General Practice, Flinders University, Adelaide, South Australia, Australia

$\dagger$ Electronic supplementary information (ESI) available: Supplemental files: ESI Table 1, Total output from the mixed model analysis. See DOI: 10.1039/c4fo00209a \$ Present address: Department of Nutrition, Institute of Basic Medical Sciences, Faculty of Medicine, University of Oslo, Norway. that may affect outcomes differently, such as those associated with CVD risk. ${ }^{2}$

Epidemiological data suggest that black and green tea may reduce the risk of both coronary heart disease and stroke by between 10 and $20 \% .^{2}$ Experimental and clinical trial data generally indicate either neutral or beneficial effects on risk factors and pathways linked to the development of CVD. ${ }^{2}$ While there is growing evidence that regular ingestion of green tea catechins with caffeine can reduce body weight and visceral fatness $^{3-9}$ there is only one study linking black tea with body weight and body fat distribution. ${ }^{10}$

In this trial we have previously demonstrated that regular ingestion of black tea can result in lower blood pressure ${ }^{11}$ and lower blood pressure variation. ${ }^{12}$ The objective of the current analysis was to assess the effects of longer-term regular consumption of black tea, over 6 months, on body weight and body fat distribution. We also assessed effects of tea on several other cardiovascular disease-related metabolic outcomes.

\section{Methods}

\subsection{Subjects and study design}

A randomized placebo-controlled double-blind 6 month parallel designed trial was performed (Fig. 1). Men and women aged 35 


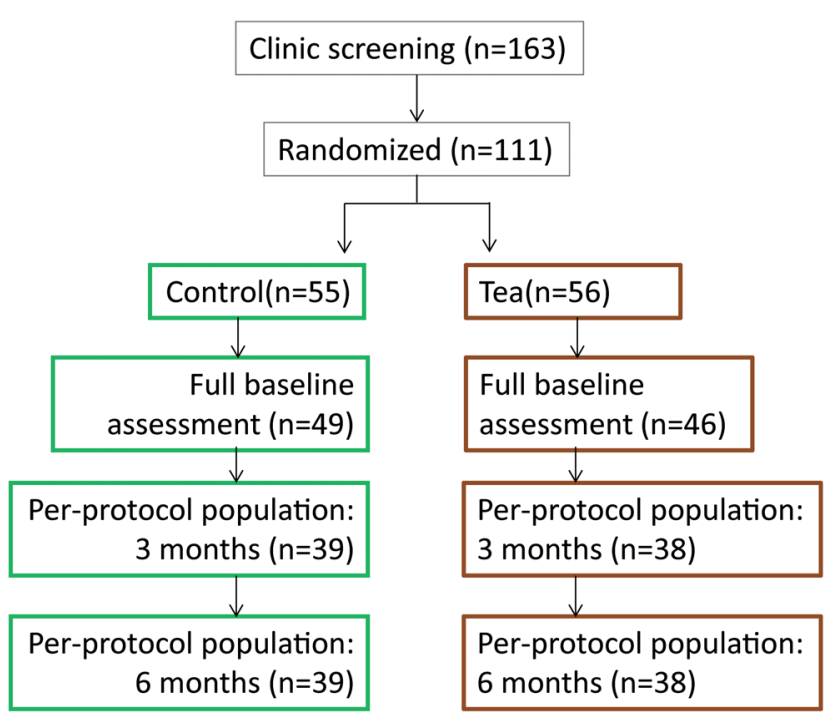

Fig. 1 Participants included at each stage of the trial.

to 75 years, who were regular tea drinkers, with a body mass index of 19 to 35 (calculated as weight in kilograms divided by height in meters squared) and a daytime ambulatory systolic blood pressure between 115 and $150 \mathrm{~mm} \mathrm{Hg}$ at screening were recruited from the general population.

Participants were enrolled in the study by a clinical trials coordinator. Prior to the 4 week run-in period volunteers were randomized ( $1: 1)$ using computer generated random numbers. Randomization codes were generated by a statistician working with Unilever Research and Development, Vlaardingen independent of study investigators. Randomization codes consisted of a four-digit number linked to the study products: either control or active tea. Study products were sachets labeled with 10 different randomly generated numbers of four digits: five linked to control and five linked to active tea. This prevented volunteers from finding out what product they were consuming by talking to each other. This also maintained blinding of researchers performing measurements during the study. A total of 120 envelopes, numbered 1 to 120 , each containing one of those 4 digit numbers, 60 linked to control and 60 linked to active tea, were produced independent of study researchers. These envelopes were then provided to the lead investigator (JMH) who was not involved directly in performing any outcome measurements. Envelopes were opened in consecutive order by the lead investigator as each participant entered into the study.

Of the 111 recruited participants 56 were randomly chosen to consume 3 cups per day of black tea. The tea was prepared from a single batch of powdered black tea provided to participants as single serve sachets. Each sachet contained black tea solids containing $429 \mathrm{mg}$ of polyphenols assessed as gallic acid equivalents measured using the folin-ciocalteu reagent with gallic acid as a standard ${ }^{13}$ and $96 \mathrm{mg}$ of caffeine (measured by HPLC). ${ }^{14}$ The contents of each sachet were dissolved in hot water prior to ingestion. 55 participants were randomly chosen to consume the placebo beverage, which was matched in flavour and caffeine content, containing no tea solids (Table 1).
Table 1 Contents, tea and placebo (per cup)

\begin{tabular}{lcc}
\hline Ingredient $(\mathrm{mg})$ & Control & Tea \\
\hline Polyphenols & 0 & 143 \\
Total caffeine & 33 & 32 \\
Tea solids & - & 498 \\
Caffeine added & 37 & - \\
Caramel colour & 90 & - \\
Tea flavour & 10 & - \\
Sugar [sucrose] & 1363 & 1403 \\
Total weight of sachet & 1500 & 1900 \\
\hline
\end{tabular}

Following randomization, participants followed a low-flavonoid diet during a 4 week run-in period and throughout the 6 month intervention. During run-in, the participants consumed 3 cups per day of regular leaf tea.

A total of 77 participants completed the trial (Fig. 1). The reasons for withdrawal in the control group were medical issues unrelated to the trial $(n=5)$, moving interstate $(n=1)$, unable to drink the beverage $(n=2)$ and personal issues $(n=8)$. The reasons for withdrawal in the tea group were incomplete baseline data $(n=1)$, medical issues unrelated to the trial $(n=6)$, moving interstate $(n=1)$, unable to drink the beverage $(n=2)$ and personal issues $(n=8)$.

\subsection{Dietary restrictions}

In order to ensure that the background flavonoid intake in both groups was less than $\sim 800-1000 \mathrm{mg}$ per day (estimated to average $\sim 500 \mathrm{mg}$ per $\mathrm{d}$ ), the intake of a limited number of high flavonoid foods and beverages (tea, coffee, red wine, chocolate, fruit juices) were restricted during a 4 week baseline period and throughout the study. Participants did not consume any additional tea during the study, they did not consume dark chocolate and were limited to less than $25 \mathrm{~g}$ per day of milk chocolate (including chocolate beverages), the wine consumed was white rather than red, the intake of fruit juices was limited to less than 4 glasses per week, and coffee intake was limited to less than 1 cup per day on average ( $<7$ cups per week). A dietitian monitored each participant's progress every 4 weeks to ensure compliance.

All procedures followed were in accordance with the ethical standards of the University of Western Australia Ethics Committee and the trial was registered at the Australian New Zealand Clinical trials Registry ACTRN12607000543482.

\subsection{Body weight and food intake}

Body weight was monitored throughout the intervention. Height was measured using a wall-mounted stadiometer. All waist and hip measurements were measured by a trained technician. A 3 day weighed food record was completed by all participants at baseline and at the end of 3 months and 6 months. 


\subsection{Plasma and urinary biomarkers}

Single biochemical measurements were performed at baseline, 3 months and 6 months. Venous blood samples were collected following a 12 hour fast from the antecubital vein of the forearm. A 24 hour urine collection was also performed. All biochemical and haematology analyses were performed in the PathWest Laboratory at Royal Perth Hospital, Western Australia. Analyses of lipids, glucose, insulin and creatinine were performed on samples stored at $-80{ }^{\circ} \mathrm{C}$, in a single batch to reduce variability using routine laboratory methods. Serum total cholesterol, HDL cholesterol and triglycerides were measured using a routine enzymatic colorimetric test with a fully automated analyser (Roche Hitachi 917; Roche Diagnostics Australia Pty Ltd., Castle Hill, NSW, Australia). Serum LDL cholesterol was calculated using the Friedewald equation. ${ }^{15}$ Serum glucose was measured using a hexokinase/G-6-PDH method (Abbott Laboratories) using a fully automated analyser (Architect c16000). The assay coefficient of variation was $2.7 \%$. Serum insulin was analysed by immunoassay (Abbott Laboratories) using a fully automated analyser (Architect c16000), with an assay coefficient of variation of $1.5 \%$. The homeostasis model assessment (HOMA) insulin sensitivity score was calculated with the following formula: [serum glucose $\left(\mathrm{mmol} \mathrm{L}^{-1}\right) \times$ serum insulin $\left.\left(\mu \mathrm{U} \mathrm{mL}{ }^{-1}\right) / 22.5\right] .{ }^{16}$ Serum and urinary creatinine were analysed using kinetic colorimetric tests (Roche, Indianapolis) with an automated analyser (Roche Hitachi 917). Estimated glomerular filtration rate was used as an indicator of renal function. Glomerular filtration rate was estimated using creatinine clearance, which was calculated in $\mathrm{mL} \mathrm{min}^{-1}$ as urinary creatinine concentration multiplied by $24 \mathrm{~h}$ urine volume, divided by plasma creatinine concentration multiplied by $24 \mathrm{~h}$ multiplied by $60 \mathrm{~min}$. Urinary 4-O-methylgallic acid (4OMGA) concentrations were measured by gas chromatography mass spectrometry according to a previously described method ${ }^{17}$ and the values were then divided by urinary creatinine concentration. The $24 \mathrm{~h}$ 4OMGA excretion is a biomarker of black tea intake and was used to assess compliance.

\subsection{Blood pressure and endothelial function}

Blood pressure measures included mean 24 hour ambulatory systolic and diastolic blood pressure and heart rate. Day time (awake), night time (asleep) and early morning blood pressure and heart rate was measured as previously described. ${ }^{11}$ Results of effects on blood pressure have previously been reported. ${ }^{11,12}$

Endothelial function measurement and analysis was performed by a trained technician, who was blinded to the treatment allocation according to a previously published method. ${ }^{18,19}$ Endothelium-dependent flow-mediated dilatation of the brachial artery (FMD) was measured following an ischemic stimulus. Analysis was carried out using a semiautomated edge detection software system. Measurements of endothelium-dependent flow-mediated dilatation of the brachial artery were performed at 30 second intervals for 4 minutes. Peak response was also assessed and was used for analysis of differences.

\subsection{Statistical analysis}

The sample size for this study was based on the primary outcome of 24 hour ambulatory systolic blood pressure. We estimated 100 participants (50 participants per group) would provide greater than $90 \%$ power at alpha 0.05 to detect a $3 \mathrm{~mm}$ $\mathrm{Hg}$ difference in 24 hour systolic blood pressure. The primary outcome from this study, blood pressure, has already been published. ${ }^{12}$ The current analysis represents an analysis of prespecified secondary outcomes for this study. The population was limited to the per protocol population which was defined as all participants who completed the 6 month study, had measurements performed for the relevant outcome and consumed at least $70 \%$ of the study product.

The effect of black tea on BMI, waist to hip circumference ratio, waist circumference, hip circumference, glucose, insulin, HOMA, triglycerides, cholesterol, HDL and LDL were analysed using mixed model analysis for longitudinal data sets i.e. each subject was measured repeatedly on the same outcome (at baseline, 3 and 6 months). The "mixed" command for linear mixed models in STATA 12 (StataCorp) with a random intercept was used treating time as a categorical parameter, with the following parameterization: $\beta 0$ month $+\beta 1$ treatment +

Table 2 Participant characteristics ${ }^{a}$

\begin{tabular}{llllll}
\hline & \multicolumn{2}{l}{ Control $(n=39)$} & & \multicolumn{2}{l}{ Tea $(n=38)$} \\
& Mean & SD & & Mean & SD \\
\hline Age & 55.4 & 9.8 & & 55.9 & 10.9 \\
Gender & 14 & & & 13 & \\
Males (n) & 25 & & 25 &
\end{tabular}

\section{Tea biomarker}

$\begin{array}{lllll}\text { 4OMGA }\left(\mu \mathrm{g} \mathrm{mmol} \mathrm{mm}^{-1}\right. & 43 & 25 & 51 & 33\end{array}$

creatinine)

Body weight and anthropometry

Waist-to-hip ratio Waist-circumference $(\mathrm{cm})$

Hip-circumference $(\mathrm{cm})$

BMI $\left(\mathrm{kg} \mathrm{m}^{-2}\right)$

Weight (kg)

$\begin{array}{rrrr}0.8 & 0.1 & 0.8 & 0.1 \\ 80.9 & 10.2 & 81.5 & 10.6 \\ 100.4 & 5.9 & 98.4 & 7.9 \\ 25.0 & 3.1 & 24.8 & 3.5 \\ 72.4 & 11.0 & 71.2 & 11.6\end{array}$

Glucose metabolism

Glucose $\left(\mathrm{mmol} \mathrm{L}^{-1}\right)$

Insulin $\left(\mathrm{mU} \mathrm{L}^{-1}\right)$

HOMA

$\begin{array}{llll}5.1 & 0.5 & 5.3 & 0.9 \\ 6.5 & 3.6 & 8.1 & 9.2 \\ 1.5 & 1.0 & 2.2 & 3.4\end{array}$

Blood lipids/lipoproteins

Triglycerids $\left(\mathrm{mmol} \mathrm{L}^{-1}\right)$

Cholesterol $\left(\mathrm{mmol} \mathrm{L}^{-1}\right)$

HDL $\left(\mathrm{mmol} \mathrm{L}^{-1}\right)$

LDL $\left(\mathrm{mmol} \mathrm{L}^{-1}\right)$

$\begin{array}{llll}1.1 & 0.6 & 1.2 & 0.5 \\ 5.2 & 0.8 & 5.1 & 0.8 \\ 1.4 & 0.1 & 1.4 & 0.1 \\ 3.3 & 0.1 & 3.1 & 0.1\end{array}$

Endothelial function

Peak FMD (\%)

$\begin{array}{llll}7.8 & 2.9 & 8.2 & 3.8\end{array}$

Max FMD diameter (mm)

$3.6 \quad 0.5$

3.5

0.5

a 4OMGA; urinary 4-O-methylgallic acid, HDL; high density lipoprotein, LDL; high density lipoprotein; FMD; flow-mediated dilatation. $p<0.005$. 
$\beta 2$ (month*treatment). Where the month*treatment was significant the difference between the treatment groups with regards to changes in the fitted mean over time are given in the text. The difference between each group at each time point was tested using model-based contrasts. All models were applied on per protocol samples. Fitted means and SE for each time and group generated by the mixed model analysis are reported. Significance was determined by $P<0.05$.

\section{Results}

\subsection{Baseline characteristics and compliance}

Patient's characteristics and baseline levels of plasma/urine variables and endothelial function are presented in Table 2. In the tea group 35 out of 38 were Caucasian and 3 of unknown ethnicity. In the control group 38 out of 39 were Caucasian and 1 was of Asian ethnicity. Compliance was confirmed by the substantially higher increase in the levels of urinary 4OMGA $(P<0.001)$ in the tea group compared to caffeine matched controls (Table 3).

\subsection{Tests for differential change over time between the intervention groups}

3.2.1 Body composition and anthropometry. Compared to controls, regular ingestion of black tea over the first 3 months inhibited gain of weight $(-0.64 \mathrm{~kg}, p=0.047)$ and BMI $(-0.22$ $\left.\mathrm{kg} \mathrm{m} \mathrm{m}^{-2}, P=0.042\right)$ and reduced waist circumference $(-1.88 \mathrm{~cm}$, $P=0.035)$ and waist-to-hip ratio $(-0.03, P=0.005)$ (Table 3$)$.

There was no significant difference between groups in the change between baseline and 6 months (Table 3). ESI Table $1 \dagger$ shows the total output from the mixed model analysis. There were no significant differences between groups with regard to changes in energy intakes throughout the study $(P=0.42$ at 3 months, $P=0.40$ at 6 months, data not shown). In addition macro-nutrient intakes were not significantly altered during the intervention.

3.2.2 Glucose metabolism and plasma lipids. There was a non-significant trend for the intake of black tea, relative to control, to result in a decrease in fasting blood glucose $(-0.2$ $\mu \mathrm{M} \mathrm{mL} L^{-1}, P=0.089$ ) at 3 months (Table 4). The magnitude of differences between the groups was smaller at 6 months. In addition, no significant differences were found over the intervention period between the tea group and control for triglycerides, cholesterol, HDL cholesterol or LDL cholesterol (Table 4). ESI Table $1 \dagger$ shows the total output from the mixed model analysis.

3.2.3 Endothelial function. Endothelial function was assessed as maximum diameter (max FMD diameter) and maximum dilation relative to vessel size (Peak FMD). No significant differences were found between the groups over time (Table 5). ESI Table $1 \dagger$ shows the total output from the mixed model analysis. In addition there were no differences between the treatment groups at 3 months or at 6 months.

\section{Discussion}

Epidemiological and intervention studies provide evidence that consumption of tea can reduce blood pressure. ${ }^{20} \mathrm{We}$ have previously reported from this study that regular black tea consumption ( 3 cups per day) for 6 months resulted in

Table 3 Tea biomarker and anthropometry measures - mixed model analysis ${ }^{a}$

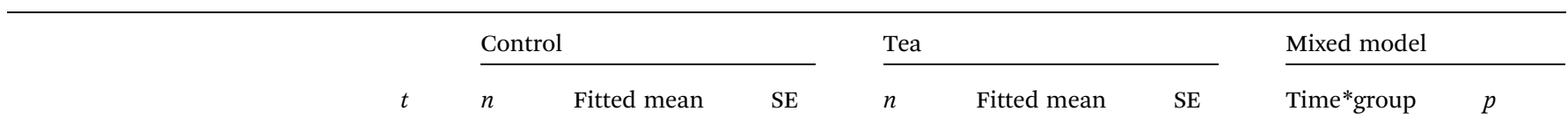

Tea biomarker

4OMGA $\mu \mathrm{g} \mathrm{mmol}^{-1}$ creatinine

$\begin{array}{lrr}0 & 39 & 43 \\ 3 & 39 & 7 \\ 6 & 39 & 7\end{array}$

\section{Anthropometry}

Waist-to-hip ratio

Waist $(\mathrm{cm})$

Hip (cm)

BMI $\left(\mathrm{kg} \mathrm{m}^{-2}\right)$

Weight (kg)

\begin{tabular}{llr}
0 & 39 & \multicolumn{1}{c}{43} \\
3 & 39 & 7 \\
6 & 39 & 7 \\
& & \\
& & \\
0 & 39 & 0.81 \\
3 & 38 & 0.82 \\
6 & 39 & 0.80 \\
0 & 39 & 80.91 \\
3 & 38 & 81.72 \\
6 & 39 & 79.78 \\
0 & 39 & 100.41 \\
3 & 38 & 99.46 \\
6 & 39 & 99.12 \\
0 & 39 & 24.97 \\
3 & 39 & 25.19 \\
6 & 39 & 25.02 \\
0 & 39 & 72.44 \\
3 & 39 & 73.08 \\
6 & 39 & 72.56
\end{tabular}

$\begin{array}{lll}4 & 38 & 51 \\ 4 & 36 & 61 \\ 4 & 37 & 70\end{array}$

51
61
70

0.02

0.02

0.02

1.69

1.69

1.69

1.08

1.09

1.08

0.52

0.52

0.52

1.80

1.80

1.80

$\begin{array}{lr}38 & 0.83 \\ 38 & 0.82 \\ 36 & 0.81 \\ 38 & 81.50 \\ 38 & 80.43 \\ 36 & 80.30 \\ 38 & 98.38 \\ 38 & 98.45 \\ 36 & 98.89 \\ 38 & 24.76 \\ 38 & 24.76 \\ 37 & 24.94 \\ 38 & 71.17 \\ 38 & 71.17 \\ 37 & 71.70\end{array}$

45

55

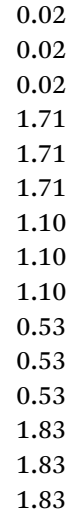

$\begin{array}{rl}-0.03 & 0.005^{*} \\ -0.02 & 0.129 \\ -1.88 & \\ -0.07 & 0.035^{*} \\ & 0.937 \\ 1.01 & 0.272 \\ 1.80 & 0.051 \\ & \\ -0.22 & 0.042^{*} \\ 0.13 & 0.225 \\ & \\ -0.64 & 0.047^{*} \\ 0.41 & 0.207\end{array}$

${ }^{a}$ Fitted means from the mixed models with SE. The $p$ values are obtained from the month*treatment interaction for 3 and 6 months respectively. Time*group corresponds to the difference in slopes (effects of time) between tea and controls. $t=$ Time (months). ${ }^{*} p$-value $<0.05$. 
Table 4 Glucose metabolism and blood lipids - mixed model analysis ${ }^{a}$

\begin{tabular}{|c|c|c|c|c|c|c|c|c|c|}
\hline & \multirow[b]{2}{*}{$t$} & \multicolumn{3}{|c|}{ Control } & \multirow[b]{2}{*}{$n$} & \multirow{2}{*}{$\frac{\text { Tea }}{\text { Fitted mean }}$} & \multirow[b]{2}{*}{ SE } & \multicolumn{2}{|l|}{ Mixed model } \\
\hline & & $n$ & Fitted mean & $\mathrm{SE}$ & & & & Time* group & $p$ \\
\hline \multicolumn{10}{|l|}{ Glucose metabolism } \\
\hline \multirow[t]{3}{*}{ Glucose $\left(\mathrm{mmol} \mathrm{L}^{-1}\right)$} & 0 & 39 & 5.09 & 0.09 & 38 & 5.31 & 0.09 & & \\
\hline & 3 & 39 & 5.10 & 0.09 & 38 & 5.12 & 0.09 & -0.20 & 0.089 \\
\hline & 6 & 39 & 5.09 & 0.09 & 37 & 5.24 & 0.09 & -0.07 & 0.572 \\
\hline \multirow[t]{3}{*}{ Insulin $\left(\mathrm{mU} \mathrm{L}^{-1}\right)$} & 0 & 39 & 6.53 & 0.82 & 38 & 8.11 & 0.83 & & \\
\hline & 3 & 39 & 6.53 & 0.82 & 38 & 6.89 & 0.83 & -1.22 & 0.238 \\
\hline & 6 & 39 & 6.15 & 0.82 & 37 & 6.80 & 0.83 & -0.94 & 0.367 \\
\hline \multirow[t]{3}{*}{ HOMA } & 0 & 39 & 1.52 & 0.26 & 38 & 2.16 & 0.27 & & \\
\hline & 3 & 39 & 1.52 & 0.26 & 38 & 1.60 & 0.27 & -0.55 & 0.177 \\
\hline & 6 & 39 & 1.42 & 0.26 & 37 & 1.62 & 0.27 & -0.43 & 0.294 \\
\hline \multicolumn{10}{|l|}{ Blood lipids/lipoproteins } \\
\hline \multirow[t]{3}{*}{ Trig $\left(\mathrm{mmol} \mathrm{L}^{-1}\right)$} & 0 & 39 & 1.12 & 0.09 & 38 & 1.15 & 0.09 & & \\
\hline & 3 & 39 & 1.03 & 0.09 & 38 & 1.08 & 0.09 & 0.02 & 0.819 \\
\hline & 6 & 39 & 1.12 & 0.09 & 37 & 1.11 & 0.09 & -0.05 & 0.566 \\
\hline \multirow[t]{3}{*}{ Total Chol $\left(\mathrm{mmol} \mathrm{L}^{-1}\right)$} & 0 & 39 & 5.24 & 0.13 & 38 & 5.08 & 0.13 & & \\
\hline & 3 & 39 & 5.30 & 0.13 & 38 & 5.27 & 0.13 & 0.13 & 0.263 \\
\hline & 6 & 39 & 5.28 & 0.13 & 37 & 5.15 & 0.13 & 0.03 & 0.764 \\
\hline \multirow[t]{3}{*}{ HDL-Chol $\left(\mathrm{mmol} \mathrm{L}^{-1}\right)$} & 0 & 39 & 1.42 & 0.05 & 38 & 1.40 & 0.05 & & \\
\hline & 3 & 39 & 1.49 & 0.05 & 38 & 1.48 & 0.05 & 0.01 & 0.760 \\
\hline & 6 & 39 & 1.47 & 0.05 & 37 & 1.46 & 0.05 & 0.01 & 0.850 \\
\hline \multirow[t]{3}{*}{ LDL-Chol $\left(\mathrm{mmol} \mathrm{L}^{-1}\right)$} & 0 & 39 & 3.30 & 0.12 & 38 & 3.14 & 0.12 & & \\
\hline & 3 & 39 & 3.34 & 0.12 & 38 & 3.29 & 0.12 & 0.11 & 0.246 \\
\hline & 6 & 39 & 3.29 & 0.12 & 37 & 3.18 & 0.12 & 0.05 & 0.609 \\
\hline
\end{tabular}

${ }^{a}$ Fitted means from the mixed models with SE. The $p$ values are obtained from the month*treatment interaction for 3 and 6 months respectively. Time*group shows the difference in effects of time between tea and controls. Tests for absolute difference between treatment groups at 3 and 6 months. Trig $=$ triglycerides; $\mathrm{Chol}=$ cholesterol.

Table 5 Endothelial function - mixed model analysis ${ }^{a}$

\begin{tabular}{|c|c|c|c|c|c|c|c|c|c|}
\hline & \multirow[b]{2}{*}{$\mathrm{t}$} & \multicolumn{3}{|c|}{ Control } & \multirow[b]{2}{*}{$n$} & \multirow{2}{*}{$\frac{\text { Tea }}{\text { Fitted mean }}$} & \multirow[b]{2}{*}{ SE } & \multicolumn{2}{|l|}{ Mixed model } \\
\hline & & $n$ & Fitted mean & $\mathrm{SE}$ & & & & Time* group & $p$ \\
\hline \multicolumn{10}{|c|}{ Endothelial function } \\
\hline \multirow[t]{3}{*}{ Peak FMD (\%) } & 0 & 39 & 3.57 & 0.08 & 38 & 3.50 & 0.08 & & \\
\hline & 3 & 39 & 3.58 & 0.08 & 38 & 3.53 & 0.08 & 0.02 & 0.244 \\
\hline & 6 & 39 & 3.62 & 0.08 & 37 & 3.53 & 0.08 & -0.02 & 0.294 \\
\hline \multirow{3}{*}{$\begin{array}{l}\text { Max FMD diam. } \\
(\mathrm{mm})\end{array}$} & 0 & 39 & 7.82 & 0.53 & 38 & 8.21 & 0.54 & & \\
\hline & 3 & 39 & 8.14 & 0.53 & 38 & 7.94 & 0.54 & -0.6 & 0.710 \\
\hline & 6 & 39 & 8.23 & 0.53 & 37 & 8.08 & 0.54 & -0.5 & 0.573 \\
\hline
\end{tabular}

${ }^{a}$ Fitted means from the mixed models with SE. The $p$ values are obtained from the month*treatment interaction for 3 and 6 months respectively. Time* group corresponds to the difference in slopes (effects of time) between tea and controls.

significantly lower systolic blood pressure, diastolic blood pressure and rate of blood pressure variation ${ }^{11,12}$ compared to control (equal amounts of caffeine but without other black tea constituents). In this analysis we demonstrate that intake of black tea, compared to control, altered the change in body weight and body fat distribution over 3 months. There was also weak evidence for improvement in glucose metabolism during this time. However, the effects on anthropometric measures during the first 3 months were not maintained over the full 6 months of the trial.
Adverse CVD risk is associated with obesity and particularly with abdominal obesity. ${ }^{21}$ In addition, waist circumference and waist to hip circumference ratio have been suggested to reflect the amount of visceral fatness..$^{22}$ Compared to the control group the tea group had a small reduction in body weight, waist circumference and waist-to-hip ratio over time, up to 3 months, suggesting that tea intake may potentially benefit visceral fat deposits. There are several reports on the effects of green tea intake on body weight and body composition..$^{3-9,23,24}$ Several of these studies were up to 12 weeks in duration. However, the only controlled clinical study on black tea and effects on 
anthropometry published so far is a 12 week double blind randomized controlled trial of 36 pre-obese Japanese adults intervened with black tea extract (BTE). ${ }^{10}$ In line with our findings, they found a significant decrease in visceral fatness as measured by computed tomography scanning. In addition they found that BMI decreased significantly in the BTE group compared to controls. ${ }^{10}$ Our results were not able to provide evidence that these potential benefits were maintained beyond 3 months, out to 6 months. The reason for this is not clear.

There are limited studies on the effect of tea on blood glucose. Intake of black tea has been reported to lower blood glucose in healthy subjects and improve scores of insulin sensitivity. ${ }^{25}$ A short-term clinical trial of healthy volunteers showed that intake of instant black tea decreased fasting blood glucose and increased fasting insulin in response to an oral glucose load. ${ }^{25} \mathrm{~A}$ clinical intervention study with oolong tea demonstrated a lowering of blood glucose in type 2 diabetic subjects. ${ }^{26}$ In vitro studies suggest a mechanism for how tea catechins can promote blood glucose regulation through induction of glucose transporter (GLUT) 4 translocation in skeletal muscle. ${ }^{27}$ Our results provide only weak evidence for a benefit of black tea on glucose concentrations. We did not find any differential effects on serum cholesterol levels between the tea group and the caffeine controlled placebo. Most human intervention studies with black tea or tea extracts have found little or no change in total serum cholesterol levels. Out of the seven randomised controlled trials on black tea only one showed cholesterol lowering effects. ${ }^{28}$

Interestingly, in the present study, the control group and the tea group had a similar pattern of increased HDL level over time. Conflicting results have been reported on the effect of black tea on HDL. Some studies find no effect of black tea intake on $\mathrm{HDL}^{29,30}$ while others have reported moderate increase in HDL when comparing a group consuming black tea to controls consuming hot water (without caffeine) ${ }^{31}$.

FMD is used to directly assess the function of the arterial wall. In the present study we did not find any effects on FMD. In the tea group, compared to control, neither Max FMD diameter nor Peak FMD was altered. This finding contradicts most other studies on black tea and FMD. ${ }^{32}$ A meta-analysis from 2011 of 9 human intervention studies where the effect of green and black tea on endothelial function had been measured by FMD concluded that moderate consumption of tea substantially and significantly enhances FMD. ${ }^{33}$ The reason for the lack of benefit in the current study may relate to the level of FMD at baseline, which was close to $8 \%$. In our hands, a healthy normolipidaemic, normotensive population has an FMD of approximately $8 \%$ (unpublished data). Thus, the FMD in the current population was essentially normal. There is evidence that it is difficult to chronically improve already normal endothelial function. ${ }^{34}$ In addition, the majority of studies reported in the literature measured the acute effect of tea on FMD, which has not been determined in the current study. Therefore, an acute-on-chronic effect of tea on FMD in the current study cannot be excluded. An improved FMD after chronic tea consumption (i.e. 4 weeks) has been demonstrated in subjects with impaired endothelial function (baseline FMD $\sim 6.0 \%$ ). ${ }^{35,36}$

\section{Limitations}

Although we found no significant differences between the groups at baseline we cannot exclude a possible regression to the mean situation for waist to hip ratio and waist circumference. However the mixed model analyses were performed including a random intercept in the model. Although decreased waist to hip ratio and waist circumference indicate decreased visceral fatness, future studies should potentially include computed tomography or magnetic resonance imaging to evaluate the effects on the different fat deposits. The risk of developing CVD in response to diet may be highly dependent on the particular genetic characteristics of an individual. ${ }^{37}$ Likewise, differences in the genetic background may dictate different response to tea intake. Variations in the genes (polymorphisms) involved in uptake, metabolism or excretion of compounds from tea are likely to be associated to differential effects of tea intake. Information on such genotypes could add another level to data interpretation.

Due to the long period of the intervention regular tea drinkers were recruited for this study. However, the amount of tea consumed daily varied between the volunteers. This made it necessary to standardise the tea intake to 3 cups of regular tea daily during the 4 week run-in period. Subsequently, subjects were randomised to the placebo or tea group. As a consequence the current study design combines the overall effect of standardised ingestion of tea in the tea group and tea withdrawal in the placebo group. It is not clear whether similar effects might be seen in a non-tea-drinking group.

\section{Conclusion}

This study demonstrates that intake of black tea may slightly affect body weight and body fat distribution as compared to caffeine-matched control beverage. The results must be interpreted cautiously since the changes in anthropometry measures were only small and the tea group did not end up having significantly lower levels of any of these parameters compared to controls at 6 months. Because tea consists of hundreds of different compounds with potential bioactivity, many yet unidentified, more work is needed to elucidate the biological activity of black tea and also to identify the bioactive compounds and their corresponding metabolites. Thus, the potential long-term consequences of regular ingestion of black tea on body weight and composition remain uncertain.

\section{Author contributions}

Dr Bøhn wrote the manuscript. Dr Bøhn and Dr Hodgson had full access to all the data in the study and take responsibility for the integrity of the data and the accuracy of the data analysis. Study concept and design: Hodgson, Puddey, Mulder, Fuchs, and Croft. Acquisition of data: Hodgson and Croft. Analysis and interpretation of data: Bøhn, Burrows, Woodman, Hodgson and Croft. Drafting of the manuscript: Bøhn and Hodgson. Statistical analysis: Bøhn, Burrows, Woodman and Hodgson. Obtained funding: Hodgson, Puddey, Mulder, Fuchs, and Croft. 
Administrative, technical, and material support: Hodgson, Puddey, Mulder, Fuchs, and Croft. Study supervision: Hodgson and Croft. All authors revised the manuscript for important intellectual content and approved the final version.

\section{Acknowledgements}

This study was supported by the National Health and Medical Research Council of Australia (grant 513744), Unilever Research and Development, and a National Health and Medical Research Council Fellowship (JMH). Funding for the study was provided by the National Health and Medical Research Council of Australia (grant 513744); and Unilever Research and Development, Vlaardingen, the Netherlands. SKB acknowledges a postdoctoral fellowship from Henning ock Throne-Holst stiftelse (Sweden). JMH was supported by an NH\&MRC Senior Research Fellowship.

\section{References}

1 J. M. Hodgson, J. M. Proudfoot, K. D. Croft, I. B. Puddey, T. A. Mori and L. J. Beilin, Comparison of the effects of black and green tea on in vitro lipoprotein oxidation in human serum, J. Sci. Food Agric., 1999, 79, 561.

2 S. K. Bøhn, N. C. Ward, J. M. Hodgson and K. D. Croft, Effects of tea and coffee on cardiovascular disease risk, Food Funct., 2012, 3, 575.

3 G. A. Cardoso, J. M. Salgado, M. C. Cesar and C. M. DonadoPestana, The effects of green tea consumption and resistance training on body composition and resting metabolic rate in overweight or obese women, J. Med. Food, 2013, 16, 120.

4 A. E. Vieira Senger, C. H. Schwanke, I. Gomes and M. G. Valle Gottlieb, Effect of green tea (Camellia sinensis) consumption on the components of metabolic syndrome in elderly, J. Nutr., Health Aging, 2012, 16, 738.

5 A. L. Brown, J. Lane, C. Holyoak, B. Nicol, A. E. Mayes and T. Dadd, Health effects of green tea catechins in overweight and obese men: a randomised controlled crossover trial, Br. J. Nutr., 2011, 106, 1880.

6 A. Basu, K. Sanchez, M. J. Leyva, M. Wu, N. M. Betts, C. E. Aston and T. J. Lyons, Green tea supplementation affects body weight, lipids, and lipid peroxidation in obese subjects with metabolic syndrome, J. Am. Coll. Nutr., 2010, 29, 31.

7 H. Wang, Y. Wen, Y. Du, X. Yan, H. Guo, J. A. Rycroft, N. Boon, E. M. Kovacs and D. J. Mela, Effects of catechin enriched green tea on body composition, Obesity, 2010, 18, 773.

8 P. Auvichayapat, M. Prapochanung, O. Tunkamnerdthai, B. O. Sripanidkulchai, N. Auvichayapat, B. Thinkhamrop, S. Kunhasura, S. Wongpratoom, S. Sinawat and P. Hongprapas, Effectiveness of green tea on weight reduction in obese Thais: A randomized, controlled trial, Physiol. Behav., 2008, 93, 486.

9 O. J. Phung, W. L. Baker, L. J. Matthews, M. Lanosa, A. Thorne and C. I. Coleman, Effect of green tea catechins with or without caffeine on anthropometric measures: a systematic review and meta-analysis, Am. J. Clin. Nutr., 2010, 91, 73.

10 K. Kubota, S. Sumi, H. Tojo, Y. Sumi-Inoue, H. Chin, Y. Oi, H. Fujita and H. Urata, Improvements of mean body mass index and body weight in preobese and overweight Japanese adults with black Chinese tea (Pu-Erh) water extract, Nutr. Res., 2011, 31, 421.

11 J. M. Hodgson, I. B. Puddey, R. J. Woodman, T. P. Mulder, D. Fuchs, K. Scott and K. D. Croft, Effects of black tea on blood pressure: a randomized controlled trial, Arch. Intern. Med., 2012, 172, 186.

12 J. M. Hodgson, K. D. Croft, R. J. Woodman, I. B. Puddey, D. Fuchs, R. Draijer, E. Lukoshkova and G. A. Head, Black tea lowers the rate of blood pressure variation: a randomized controlled trial, Am. J. Clin. Nutr., 2013, 97, 943.

13 V. L. Singleton, R. Orthofer and R. M. Lamuela-Raventos, Analysis of total phenols and other oxidation substrates and antioxidants by means of Folin-Ciocalteu reagent, Methods Enzymol., 1999, 299, 152.

$14 \mathrm{~S}$. Kuhr and U. Engelhardt, Determination of flavanols, theogallin, gallic acid and caffeine in tea using HPLC, $Z$. Lebensm.-Unters. Forsch., 1991, 192, 526.

15 W. T. Friedewald, R. I. Levy and D. S. Fredrickson, Estimation of the concentration of low-density lipoprotein cholesterol in plasma, without use of the preparative ultracentrifuge, Clin. Chem., 1972, 18, 499.

16 D. R. Matthews, J. P. Hosker, A. S. Rudenski, B. A. Naylor, D. F. Treacher and R. C. Turner, Homeostasis model assessment: insulin resistance and beta-cell function from fasting plasma glucose and insulin concentrations in man, Diabetologia, 1985, 28, 412.

17 J. M. Hodgson, I. B. Puddey, K. D. Croft, V. Burke, T. A. Mori, R. A. Caccetta and L. J. Beilin, Acute effects of ingestion of black and green tea on lipoprotein oxidation, Am. J. Clin. Nutr., 2000, 71, 1103.

18 G. Dogra, L. Rich, K. Stanton and G. F. Watts, Endotheliumdependent and independent vasodilation studies at normoglycaemia in type I diabetes mellitus with and without microalbuminuria, Diabetologia, 2001, 44, 593.

19 R. J. Woodman, D. A. Playford, G. F. Watts, C. Cheetham, C. Reed, R. R. Taylor, I. B. Puddey, L. J. Beilin, V. Burke, T. A. Mori and D. Green, Improved analysis of brachial artery ultrasound using a novel edge-detection software system, J. Appl. Physiol., 2001, 91, 929.

20 A. Deka and J. A. Vita, Tea and cardiovascular disease, Pharmacol. Res., 2011, 64, 136.

21 R. H. Eckel, S. M. Grundy and P. Z. Zimmet, The metabolic syndrome, Lancet, 2005, 365, 1415.

22 F. F. Ribeiro-Filho, A. N. Faria, S. Azjen, M. T. Zanella and S. R. Ferreira, Methods of estimation of visceral fat: advantages of ultrasonography, Obes. Res., 2003, 11, 1488.

23 A. L. Brown, J. Lane, J. Coverly, J. Stocks, S. Jackson, A. Stephen, L. Bluck, A. Coward and H. Hendrickx, Effects of dietary supplementation with the green tea polyphenol epigallocatechin-3-gallate on insulin resistance and associated metabolic risk factors: randomized controlled trial, Br. J. Nutr., 2009, 101, 886. 
24 C. H. Hsu, T. H. Tsai, Y. H. Kao, K. C. Hwang, T. Y. Tseng and P. Chou, Effect of green tea extract on obese women: a randomized, double-blind, placebo-controlled clinical trial, Clin. Nutr., 2008, 27, 363.

25 J. A. Bryans, P. A. Judd and P. R. Ellis, The effect of consuming instant black tea on postprandial plasma glucose and insulin concentrations in healthy humans, J. Am. Coll. Nutr., 2007, 26, 471.

26 K. Hosoda, M. F. Wang, M. L. Liao, C. K. Chuang, M. Iha, B. Clevidence and S. Yamamoto, Antihyperglycemic effect of oolong tea in type 2 diabetes, Diabetes Care, 2003, 26, 1714.

27 M. Ueda, S. Nishiumi, H. Nagayasu, I. Fukuda, K. Yoshida and H. Ashida, Epigallocatechin gallate promotes GLUT4 translocation in skeletal muscle, Biochem. Biophys. Res. Commun., 2008, 377, 286.

28 J. M. Hodgson, Tea flavonoids and cardiovascular disease, Asia Pac. J. Clin. Nutr., 2008, 17(suppl 1), 288.

29 H. M. Princen, D. W. van, R. Buytenhek, C. Blonk, L. B. Tijburg, J. A. Langius, A. E. Meinders and H. Pijl, No effect of consumption of green and black tea on plasma lipid and antioxidant levels and on LDL oxidation in smokers, Arterioscler., Thromb., Vasc. Biol., 1998, 18, 833.

30 M. J. Davies, J. T. Judd, D. J. Baer, B. A. Clevidence, D. R. Paul, A. J. Edwards, S. A. Wiseman, R. A. Muesing and S. C. Chen, Black tea consumption reduces total and LDL cholesterol in mildly hypercholesterolemic adults, J. Nutr., 2003, 133, 3298 S.
31 T. Bahorun, A. Luximon-Ramma, V. S. Neergheen-Bhujun, T. K. Gunness, K. Googoolye, C. Auger, A. Crozier and O. I. Aruoma, The effect of black tea on risk factors of cardiovascular disease in a normal population, Prev. Med., 2012, 54(suppl), S98.

32 D. Grassi, T. P. Mulder, R. Draijer, G. Desideri, H. O. Molhuizen and C. Ferri, Black tea consumption dose-dependently improves flow-mediated dilation in healthy males, J. Hypertens., 2009, 27, 774.

33 R. T. Ras, P. L. Zock and R. Draijer, Tea consumption enhances endothelial-dependent vasodilation; a metaanalysis, PLoS One, 2011, 6, e16974.

34 S. H. Li, X. X. Liu, Y. Y. Bai, X. J. Wang, K. Sun, J. Z. Chen and R. T. Hui, Effect of oral isoflavone supplementation on vascular endothelial function in postmenopausal women: a meta-analysis of randomized placebo-controlled trials, Am. J. Clin. Nutr., 2010, 91, 480.

35 J. M. Hodgson, I. B. Puddey, V. Burke, G. F. Watts and L. J. Beilin, Regular ingestion of black tea improves brachial artery vasodilator function, Clin. Sci., 2002, 102, 195.

36 S. J. Duffy, J. F. Keaney, Jr, M. Holbrook, N. Gokce, P. L. Swerdloff, B. Frei and J. A. Vita, Short-and long-term black tea consumption reverses endothelial dysfunction in patients with coronary artery disease, Circulation, 2001, 104, 151.

37 D. Corella and J. M. Ordovas, Nutrigenomics in cardiovascular medicine, Circ.: Cardiovasc. Genet., 2009, 2, 637. 